\title{
Cribriform Plate Anatomical Variations: A Computed Tomography Study
}

\author{
Carlos Romualdo Rueff-Barroso, ${ }^{1}$ Tânia Mara de Sá Ferreira, ${ }^{2}$ Caroline Fernandes-Santos, ${ }^{3}$ Valéria Paula Sassoli Fazan ${ }^{4}$ \\ ${ }^{1}$ Department of Morphology, Health Sciences Center, Federal University of Espírito Santo (UFES), Vitória, ES, Brazil \\ ${ }^{2}$ Physiotherapist; São Paulo, SP, Brazil \\ ${ }^{3}$ Department of Basic Sciences, Federal Fluminense University, Nova Friburgo, RJ, Brazil \\ ${ }^{4}$ Department of Surgery and Anatomy, School of Medicine of Ribeirão Preto (FMRP), University of São Paulo (USP), Ribeirão Preto, SP, Brazil
}

Disclose and conflicts of interest: none to be declared by all authors

\begin{abstract}
Introduction: knowledge of anatomy and anatomical variations of the ethmoid bone, particularly the cribriform plate, is of great importance for otolaryngologists and neurosurgeons, as one of the complications that can occur during surgery of the nasal cavity or anterior cranial fossa is cerebrospinal fluid (CSF) fistula or nasal liquorrhea, resulting from perforation of the cribriform plate. We aimed to analyze the anatomical variations of the cribriform plate using computed tomography images of the skull.

Materials and Methods: one hundred and Twenty-five exam images were analyzed, of which 50 were from men and 75 from women. The evaluated parameters were the symmetry of the cribriform plate and the horizontal, oblique, or vertical layout of the lamellae.

Results: the cribriform plate was shown to be asymmetrical in $82 \%$ of men and $20 \%$ of women $(p<0.0001)$. Regarding the asymmetry of the cribriform plate, $98 \%$ of men had a higher left lamina, while $73 \%$ of women had this same pattern $(p=0.02)$. The vertical positioning of the lamella was the most frequent finding in both men and women ( $48 \%$ and $44 \%$, respectively). Conclusions: Women have a more symmetrical arrangement of the cribriform plate. When there is asymmetry, the left portion is higher in both genders. The vertical arrangement of the lamellae is the most common finding between both genders.

Keywords: Anatomical variations, Cribriform plate, Computed tomography, Cerebrospinal fluid rhinorrhea.
\end{abstract}

\section{Introduction}

Statistical and physiological criteria are used to define anatomical normality: normal is the most frequent structure and best adapted for good performance. Anatomical variations are small normal morphological deviations from normal, which do not disturb function. ${ }^{1}$

The ethmoid is a light and spongy bone, located in the anterior part of the skull base, between the two orbital cavities. It has four parts: a horizontal one, called a cribriform plate, which forms part of the base of the skull; a perpendicular plate that contributes to the formation of the nasal septum; and two lateral masses or labyrinths. ${ }^{2-5}$ The upper wall of the nasal cavity is formed mainly by the cribriform plate. ${ }^{7}$ The foramina of the cribriform plate are perforations that give passage to the olfactory nerve filaments which are surrounded by dura mater and arachnoid-sheaths. It belongs to the olfactory region of the nasal cavities and it is where the olfactory bulb lays on inside the cranial cavity. ${ }^{4,5,7}$ The lateral lamella of the cribriform plate is a part of thin bone located between the cribriform plate and the ethmoidal fovea. At the junction of the ethmoidal fovea with this lamella is the canal for the anterior ethmoidal artery, that is at risk of injury in endoscopic sinus surgery. ${ }^{8}$ The ethmoid bone is prone to develop morphological variations in its bone structures during its development and pneumatization process $^{9,10}$. Ethmoidal cells are present at birth and continue to grow until puberty. ${ }^{9,10}$ Also,the nasal cavity is shown to continue to increase with age..$^{11,12}$

Knowledge of anatomy and anatomical variations of the ethmoid bone, particularly the cribriform plate, is of great importance for otolaryngologists and neurosurgeons, as one of the complications that can occur during surgery of the nasal cavity or anterior cranial fossa is cerebrospinal fluid (CSF) fistula or nasal liquorrhea, resulting from perforation of the cribriform plate. Despite the literature presenting several computed tomography investigations of the nasal cavity anatomical variations, ${ }^{13-15}$ investigations of the cribriform plate variations are still scanty. We aimed to analyze the presence of anatomical variations of the cribriform plate using images of computed tomography of the skull and their frequency between genders. 


\section{Materials and Methods}

A retrospective evaluation of sinuses examinations, obtained with computed tomography (CT) scan file, carried out in a period of 3 years $(2005-2008)$, at the Radiology Service of the Hospital Universitário Pedro Ernesto (HUPE), of the State University of Rio de Janeiro, was carried out. Only exams of patients without known ear, nose, or throat disease, were used in this study. Also, we foccused exclusevely on aspects of the normal bone anatomy of the images in the archive files. This study was authorized by the head of the radiology department and by the head of the computed tomography sector at HUPE.

In total, $125 \mathrm{CT}$ exam images were evaluated, 50 of which from male and 75 female patients. The examinations were performed on a GE HiSpeed helical tomography equipment (General Electric, New Jersey, USA), with $2 \mathrm{~mm}$ thick cuts in the coronal plane. All analyzes were performed on coronal images, taking the level of the ostium-meatus complexes as an anatomical reference point.

The analysis of the morphological aspects of the cribriform plate and the lamellae in computed tomography images were performed under the guidance of two radiologists. For the study, the cribriform plate seen in the coronal sections was divided into two portions, one at the right $(\mathrm{R})$ and the other to the left (L). The ethmoidal crest was used as the boundary of these portions (Figure 1).

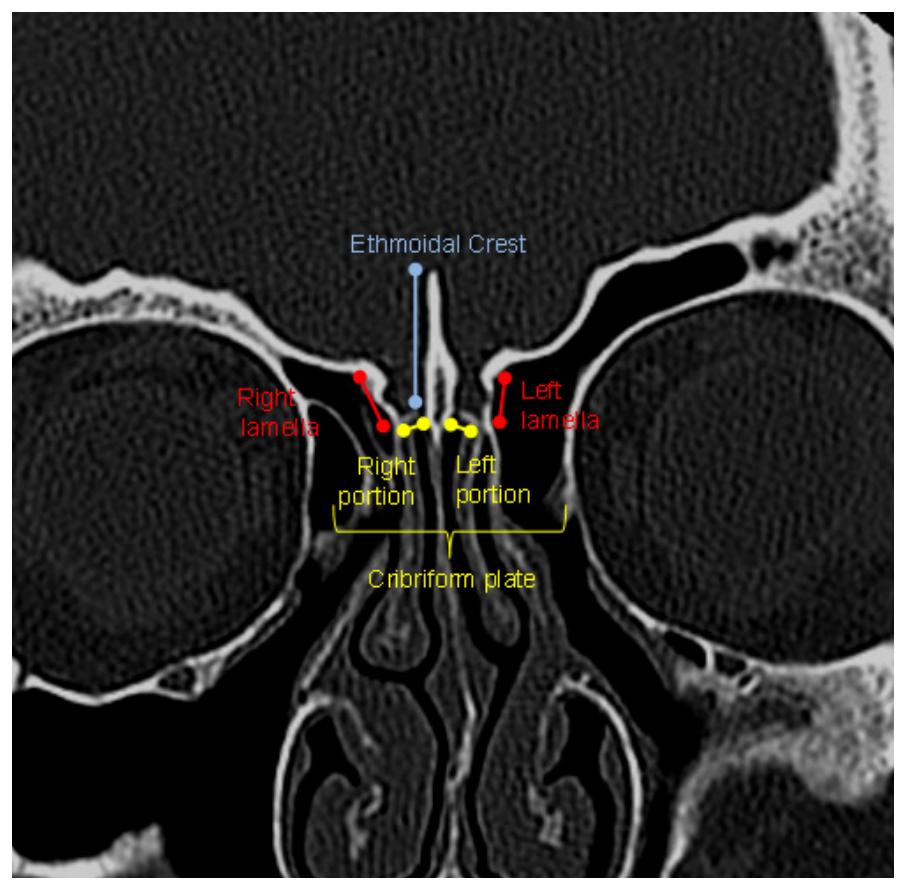

Figure 1. Computed tomography image of the ethmoidal sinus in a coronal section showing the position of the ethmoidal crest, the right and left lamellae and the cribriform plate divided into right and left portions.

The positions of the right and left portions were classified as symmetrical (when they were arranged in the same horizontal plane) or asymmetrical (portions positioned in different horizontal planes) (Figure 2).
When an asymmetric disposition was present, we indicated which side was positioned at a higher level concerning the other $(\mathrm{L}>\mathrm{R}$, upper left side higher; $\mathrm{R}>\mathrm{L}$, upper right side higher).
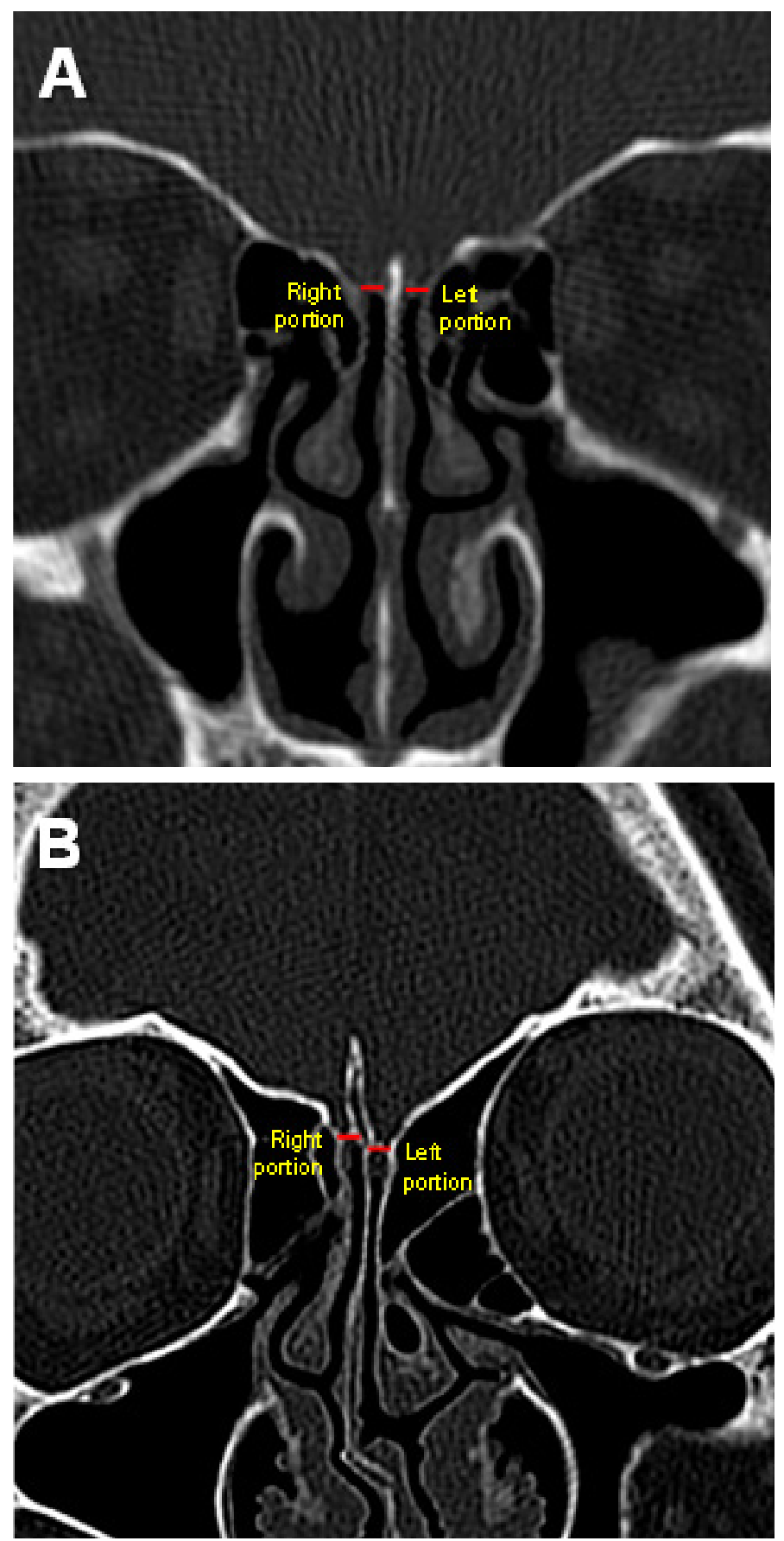

Figure 2. (A) Computed tomography image of the ethmoidal sinus in a coronal section showing the symmetrical positioning of the portions of the cribriform plates. (B) Computed tomography image of the sinuses in coronal section showing the asymmetric positioning of the portions of the cribriform plates. In this example, we have the $R>L$ situation.

The lamellae were classified into three categories, based on their angle with the axis of the cribriform plate, in the coronal plane. The angle increases as the lamella approach, superiorly, the axis of the ethmoidal crest. Thus, we consider three types of lamellae: horizontal (angle between 0 and $20^{\circ}$ ), oblique (angle 
between $20^{\circ}$ and $70^{\circ}$ ), or vertical (angle between $70^{\circ}$ and $90^{\circ}$ ) lamellae (Figure 3 ).

For the statistical analysis, age is presented as mean \pm standard error of the mean. The other data are presented in the form of frequency (\% of total). For the analysis of the difference between genders, the parameters referring to the cribriform plate (symmetry/asymmetry and upper asymmetric side) the Fisher's exact test (contingency table 2x2) was used, while data regarding the angle of the lamella were analyzed by the Chi-square test (3x2 contingency table). A p <0.05 was considered statistically significant. Statistical analysis was performed using the GraphPad Prism 4.0 software (CA, USA).

\section{Results}

The mean age of the patients analyzed was $32 \pm 3$ for men and $43 \pm 2$ for women.

The cribriform plate presented asymmetry in $82 \%$ of men (Figure 4). The opposite was seen in women: $80 \%$ presented symmetry, with the proportion of symmetry/asymmetry being different between genders $(\mathrm{p}<0.0001)$ (Figure 4$)$. In relation to patients with asymmetry of the cribriform plate, $98 \%$ of men presented a higher left portion $(\mathrm{L}>\mathrm{R})$, while only $73 \%$ of women presented this same pattern (Figure 5). The proportion between parameters $\mathrm{L}>\mathrm{R}$ and $\mathrm{R}>\mathrm{L}$ was different between genders $(p<0.02)$ (Figure 5). The vertical positioning of the lamella was the most frequent finding in both men and women (48\% and $44 \%$, respectively) (Figure 6). Oblique lamellae were also frequent among men (40\%), however in women, the obliquely or horizontally arranged lamellae presented a similar frequency (29\% and 27\%, respectively) (Figure $6)$. The Chi-square test confirms this difference in the proportion of types of lamella angulation between genders $(\mathrm{p}<0.04)$.

\section{Discussion}

Perforation of the cribriform plate during surgeries of the nasal cavity or anterior cranial fossa is characterized by the presence of cerebrospinal fluid (CSF) in the nasal cavity and occurs due to a bony and dural opening, alowing the subarachnoid space and upper airways to communicate. ${ }^{16}$ The etiology of CSF fistula can be due to traumatic and nontraumatic conditions, ${ }^{6,17}$ with the latter presenting a low incidence. ${ }^{18}$ Most iatrogenic fistulas occur due to accidental trauma of the cribriform plate. ${ }^{16-18}$ It is present in about $30 \%$ of skull-base surgeries. ${ }^{16-19}$ The areas of greatest risk for causing CSF leak are the ethmoid cribriform plate and the ethmoid fovea. ${ }^{19,20}$

The distance between the ethmoidal fovea and the cribriform plate varies between 4 and $16 \mathrm{~mm}$ and, in most cases, between 4 and $7 \mathrm{~mm}$. The knowledge of this distance is of great importance during endonasal micro-surgeries, since the relationship between the ethmoidal cells and the anterior cranial fossa will be
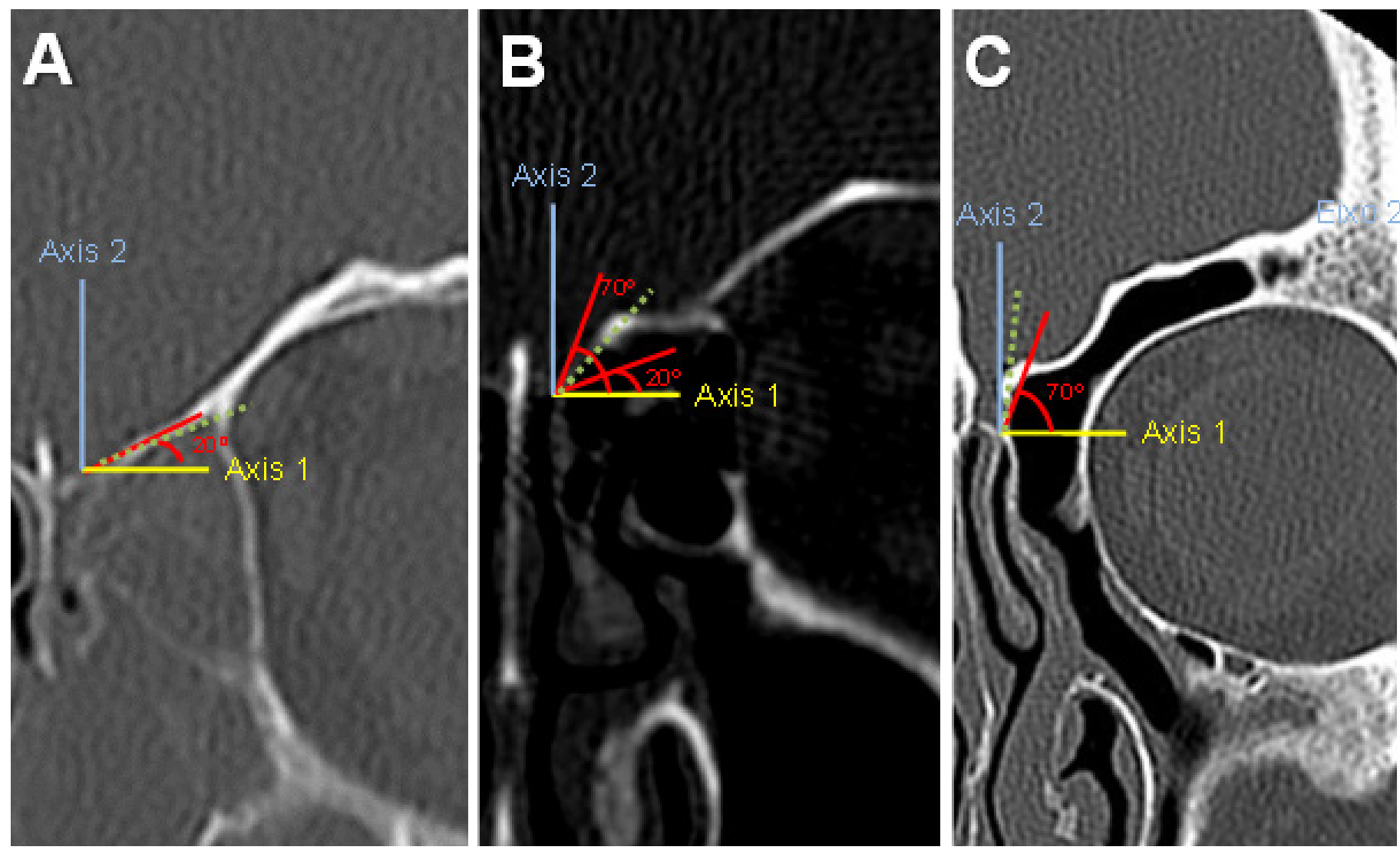

Figure 3. Computed tomography images of the ethmoidal sinus in coronal sections demonstrating the classification of the lamellae according to the angle concerning the axis of the ethmoidal crest. Horizontal lamella (A), Oblique lamella (B) and Vertical lamella (C). 


\section{Percentage of Symetric Cribriform Plates}

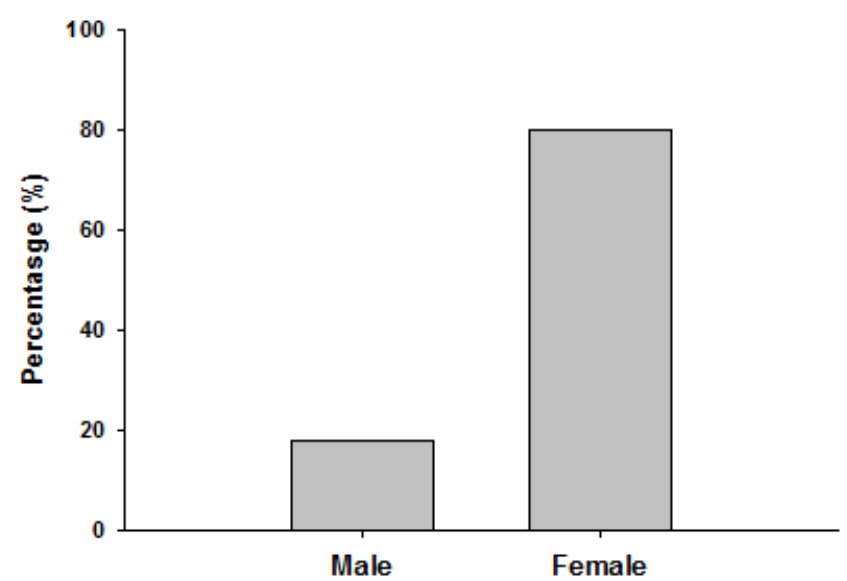

Percentage of Asymmetric Cribriform Plates

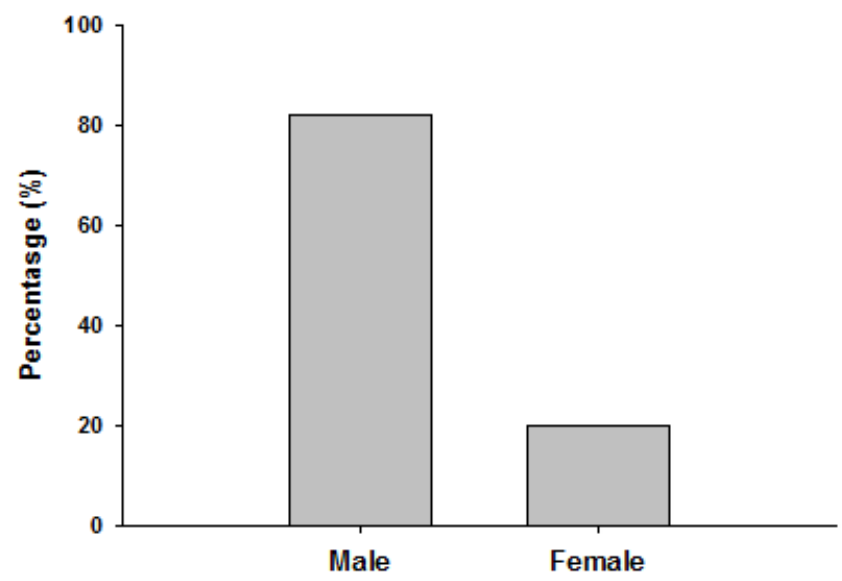

Figure 4. Percentage of symmetric and asymmetric positioning of the portions of the cribriform plates, according to gender.

R > L Cribriform Plate

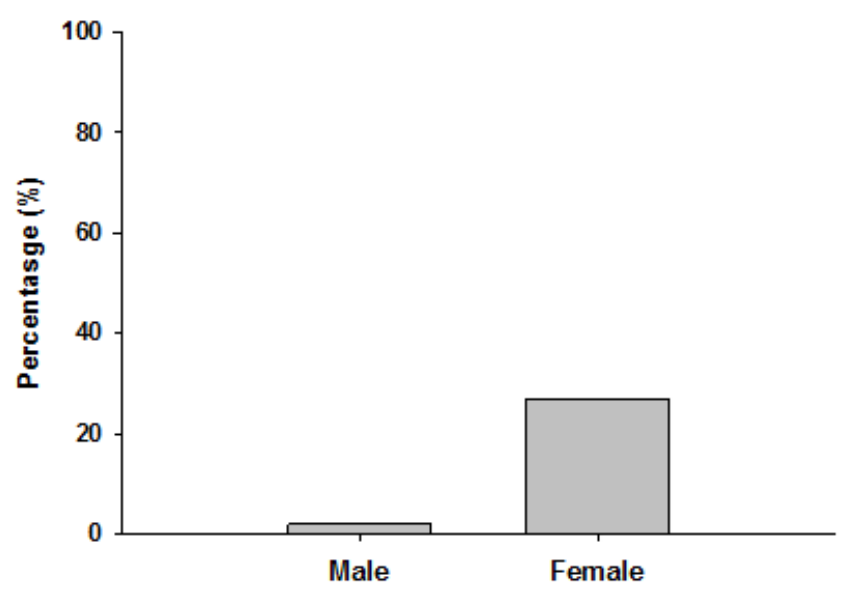

L > R Cribriform Plate

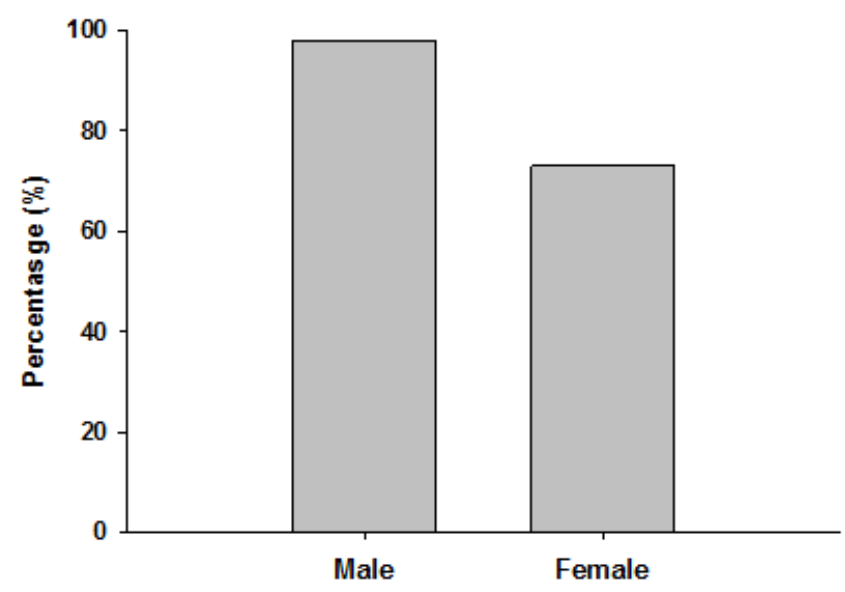

Figure 5. Percentage of asymmetric cribriform plates, considering upper right side blade $(R>L)$ or upper left side blade ( $\mathrm{L}>\mathrm{R}$ ), according to gender.

Cribriform Lamella Positioning

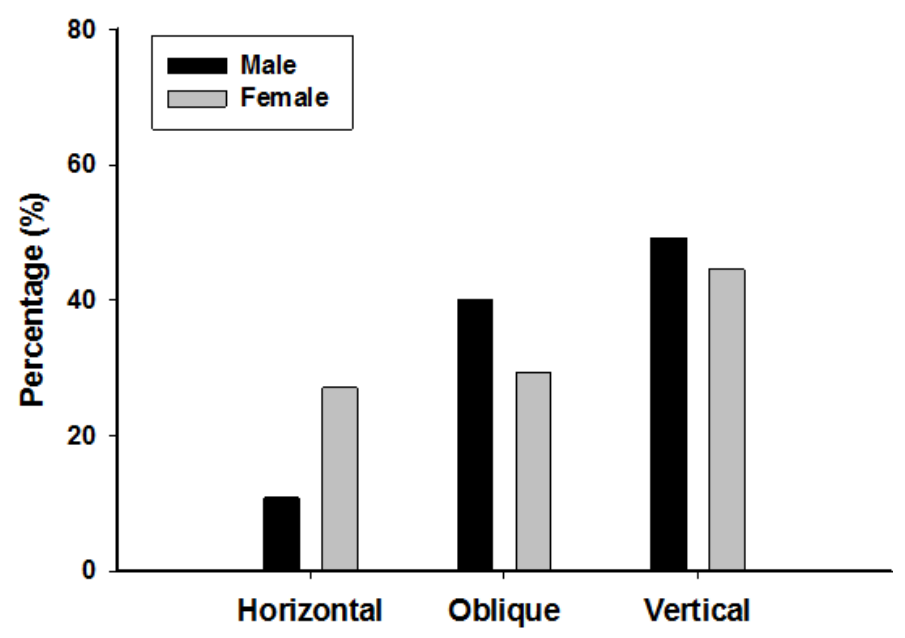

Figure 6. Percentage of cribriform lamellae according to the classification into horizontal, oblique, or vertical, in both genders. bigger as the distance between the ethmoidal fovea and the cribriform plate increases. ${ }^{21}$

The distance between the highest level of the ethmoidal labyrinth and the cribriform plate varies between 0.6 and $11.7 \mathrm{~mm}$, and the distance between the floor of the nasal cavity and the cribriform plate, varies between 38 and $52 \mathrm{~mm}$. These anatomical variations are also important to be noticed during surgeries, as they can increase the risk of complications. ${ }^{21}$

When analyzing Figure 4, we observe that the asymmetry of the cribriform plate occurs in a greater proportion in men compared to women. This finding suggests that the determination of the positioning of the portions of the cribriform plate would be influenced by gender, where women have a more regular, symmetrical pattern in the arrangement of portions of the cribriform plate when compared to men. However, we suggest that these differences may be due to sexual dimorphism in the skull. 
Cranial weight and capacity are greater in men than in women..$^{22,23}$ The female skull is characterized by less developed structures, being anatomical accidents smaller and smoother. ${ }^{22,23}$ Differentiated craniofacial growth between genders are verified in childhood, puberty and adulthood, due to the growth of facial soft tissues..$^{22,23}$

Regarding the asymmetric positioning of the portions of the cribriform plate, we also observed that, whenever there was an asymmetric pattern, the higher left portion $(L>R)$ was the most frequent finding in both men and women. We can infer that this is an important finding for surgical access planning.

The analysis of the lamellae based on the angulation with the axis of the cribriform plate in the coronal plane showed that the verticalized lamella is the most frequent finding in both genders. The low position of the ethmoidal fovea, the cribriform lamina and its lateral lamella is a dangerous anatomical variation, which can be easily accidentaly perforated by the surgeon when not aware of the possibility., ${ }^{3,719-21}$

\section{Conclusion}

This investigation showed that women presented a more symmetrical arrangement of the portions of the cribriform plate while men presented an asymmetrical pattern. Whenever asymmetry occurred, the left portion was higher in both genders. The vertical arrangement of the lamellae was also the most common finding among the genders.

Variations in the anatomical pattern of the cribriform plate are very common and are well observed by computed tomography images of the skull. However, it is not possible to attribute with certainty the dominant influence of gender on the changes. Further investigations are needed to assess and correlate anatomical findings and genders with studies of anthropometric measurements of the skull.

\section{Aknowledgements}

We deeply thank Professor José Fernando Cardona Zanier of the Department of Internal Medicine Radiology Service of Hospital Universitário Pedro Ernesto - UERJ, for all the technical assistance and feasibility to carry out this work.

\section{References}

1. Żytkowski A, Tubbs RS, Iwanaga J, E, Polguj M, Wysiadecki G. Anatomical normality and variability: Historical perspective and methodological considerations. Translational Research in Anatomy 2021,23:100105. doi: 10.1016/j.tria.2020.100105.

2. Jankowski R. Revisiting human nose anatomy: Phylogenic and ontogenic perspectives. Laryngoscope 2011;121. doi: 10.1002/ lary. 21368

3. Ogle OE, Weinstock RJ, Friedman E. Surgical anatomy of the nasal cavity and paranasal sinuses. Oral Maxillofac Surg Clin North Am 2012;24:155-166. doi: 10.1016/j.coms.2012.01.011.

4. Cauwenberge PV, Sys L, De Belder T, Watelet JB. Anatomy and physiology of the nose and the paranasal sinuses. Immunol Allergy Clin North Am 2004;24:1-17. doi: 10.1016/S0889-8561(03)00107-3.

5. Baroody FM. Nasal and paranasal sinus anatomy and physiology. Clin Allergy Immunol 2007;19:1-21.

6. Tamakawa Y, Hanaffe WN. Cerebrospinal Fluid Rhinorrhea: The Significance of an Air-Fluid Level in the Sphenoid Sinus. Radiology 1980;13:101-103.

7. Terrier F, Weber W, Ruefenacht D, Porcellini B. Anatomy of the Ethmoid: CT, Endoscopic, and Macroscopic. AJR Am J Roentgenol 1985;144:493-500. doi: 10.2214/ajr.144.3.493.

8. Abdullah B, Lim EH, Husain S, Snidvongs K, Wang DY. Anatomical variations of anterior ethmoidal artery and their significance in endoscopic sinus surgery: a systematic review. Surg Radiol Anat 2019;41:491-499. doi:10.1007/s00276-018-2165-3.

9. Wolf G, Anderhuber W, Kuhn F. Development of the paranasal sinuses in children: implications for paranasal sinus surgery. Ann Otol Rhinol Laryngol 1993;102:705-711. doi: 10.1177/000348949310200911.

10. O'Tuama LA, Swanson MS. Development of paranasal and mastoid sinuses: a computed tomographic pilot study. J Child Neurol 1986;1:46-9. doi: 10.1177/088307388600100107.

11. Loftus PA, Wise SK, Nieto D, Panella N, Aiken A, DelGaudio JM. Intranasal volume increases with age: Computed tomography volumetric analysis in adults. Laryngoscope 2016;126:2212-2215. doi: 10.1002/lary.26064.

12. Ganjaei KG, Soler ZM, Mappus ED, Worley ML, Rowan NR, Garcia GJM, Matthews LJ, Dubno JR, Eckert MA, Schlosser RJ. Radiologic changes in the aging nasal cavity. Rhinology 2019;57:117-124. doi:
10.4193/Rhin18.096.

13. Pérez-Piñas JS, Carmona A, Catalina-Herrera CJ, JiménezCastellanos J. Anatomical variations in the human paranasal sinus region studied by CT. J Anat 2000;197:221-227. doi: 10.1046/j.14697580.2000.19720221.x.

14. Kayalioglu G, Oyar O, Govsa F. Nasal cavity and paranasal sinus bony variations: a computed tomographic study. Rhinology 2000;38:108-113.

15. Nautiyal A, Narayanan A, Mitra D, Honnegowda TM, Sivakumar. Computed Tomographic Study of Remarkable Anatomic Variations in Paranasal Sinus Region and their Clinical Importance - A Retrospective Study. Ann Maxillofac Surg 2020;10:422-428. doi: 10.4103/ams.ams_192_19.

16. Mishra SK, Mathew GA, Paul RR, Asif SK, John M, Varghese AM, Kurien M. Endoscopic Repair of CSF Rhinorrhea: An Institutional Experience. Iran J Otorhinolaryngol 2016;28:39-43.

17. Daele JJM, Goffart Y, Machiels S. Traumatic, iatrogenic, and spontaneous cerebrospinal fluid (CSF) leak: endoscopic repair. B-ENT 2011;7 Suppl 17:47-60.

18. Wise SK, Schlosser RJ. Evaluation of spontaneous nasal cerebrospinal fluid leaks. Curr Opin Otolaryngol Head Neck Surg 2007;15:28-34. doi: 10.1097/M00.0b013e328011bc76

19. Tabaee A, Kassenoff TL, Kacker A, Anand VK. The Efficacy of Computer Assisted Surgery in the Endoscopic Management of Cerebrospinal Fluid Rhinorrhea. Otolaryngol Head Neck Surg 2005;133:936-943.

20. White DR, Dubin MG, Senior BA. Endoscopic Repair of Cerebrospinal Fluid Leaks After Neurosurgical Procedures. Am J Otolaryngol 2003;24:213-216. doi: 10.1016/s0196-0709(03)00031-0.

21. Onerci TM, Ayhan K, Oğretmenoğlu O. Two Consecutive Cases of Cerebrospinal Fluid Rhinorrhea After Septoplasty Operation. Am J Otolaryngol 2004;25:354-356. doi: 10.1016/j.amjoto.2004.03.005.

22. Spradley MK, Jantz RL. Sex estimation in forensic anthropology: skull versus postcranial elements. J Forensic Sci 2011;56:289-296. doi: $10.1111 / j .1556-4029.2010 .01635 . x$.

23. Kimmerle EH, Ross A, Slice D. Sexual dimorphism in America: geometric morphometric analysis of the craniofacial region. J Forensic Sci 2008;53:54-57. doi: 10.1111/j.1556-4029.2007.00627.x. 


\section{Mini Curriculum and Author's Contribution}

1.Carlos Romualdo Rueff-Barroso: PT; Ph.D. Contribution: Study desing, data collectoin, data interpreatation, manscript preparation and literature search.

2. Tânia Mara de Sá Ferreira: PT. Contribution: Data collection, manuscript preparation and literature search.

3. Caroline Fernandes-Santos: BSc; Ph.D. Contribution: Data collection, statistical analysis and data interpretation.

4. Valéria Paula Sassoli Fazan: M.D.; Ph.D. Contribution: Data interpretation, critically revising the manuscript, approval of the final version. ORCID: 0000-0003-1293-5308. 